\title{
Development of an item pool for a questionnaire on the psychosocial consequences of hypertension labelling
}

\author{
János Valery Gyuricza ${ }^{1 *}$ DD, Ana Flávia Pires Lucas d'Oliveira', Lucas Bastos Marcondes Machado² and \\ John Brodersen ${ }^{3}$
}

\begin{abstract}
Background: Hypertension is the most prevalent risk factor for cardiovascular disease globally. Roughly one-third of the adult population has hypertension. However, most people diagnosed with hypertension do not benefit from blood pressure control with pharmacologic interventions: they are overdiagnosed and overtreated and might experience negative psychosocial consequences of being labelled. These consequences are relevant outcomes that need to be assessed and validly measured to identify all benefits and harms related to interventions designed to prevent cardiovascular disease.
\end{abstract}

Objectives: To develop a pool of items with high content validity for a draft version of a condition-specific questionnaire to measure the psychosocial consequences of being labelled with mild hypertension.

Methods: We selected relevant items from existing Consequences of Screening (COS) questionnaires. These items belonged to two groups: COS core items and potential condition-specific items. All items were originally in Danish and were translated into Brazilian Portuguese using the dual-panel method. Individual and group interviews were conducted with people with mild hypertension and low risk for cardiovascular disease, and were designed to test the translated items for face and content validity and were also used to generate new relevant items. Structured individual interviews were conducted to categorise all the items into domains.

Results: The Brazilian Portuguese dual-panel translation of both groups of items was found to be relevant for adults diagnosed with hypertension. We generated 52 new items to achieve high content validity. The result was a set of 132 items divided into 22 domains in 2 parts. Part I was directed at the general population, whereas part II was directed only at people diagnosed with hypertension and it consisted of 38 items in 8 domains. Twelve items remained as single items. High content validity was achieved with the pool of 132 items divided into 22 domains in 2 parts.

Discussion: High content validity was achieved for a condition-specific questionnaire measuring the psychosocial consequences of being labelled with mild hypertension. This instrument encompassed 132 items divided into 22 domains in 2 parts. Thereby, a draft of the Consequneces of Hypertension questionnaire $(\mathrm{COH})$ was developed. The psychometric properties of this questionnaire will be discussed in a diferent paper.

Keywords: Psychosocial consequences, Patient-reported outcome measure, Hypertension

\footnotetext{
* Correspondence: jvgyuricza@usp.br

'Departamento de Medicina Preventiva, Faculdade de Medicina da

Universidade de São Paulo, Av. Dr Arnaldo, 45520 andar. CEP, São Paulo, SP

01246-903, Brazil

Full list of author information is available at the end of the article
} 


\section{Background}

Hypertension is the most prevalent risk factor related to the development of cardiovascular disease. It is in most cases an asymptomatic condition, and its diagnosis is dependent on preventive strategies directed at the general population or at patients visiting the doctor. Currently, hypertension is defined in Brazil as blood pressure averages above 140/90 $\mathrm{mmHg}$ [1], divided in three stages, as shown in Table 1.

When implementing preventive health strategies, both intended benefits and unintended harms are expected. These two sides can be counterweighed in various ways, but benefits and harms are multidimensional [2] and are not always easily understood and quantified [3]. In the case of hypertension, the benefits in terms of morbidity and mortality are clear to those patients in the moderate and high-risk groups (moderate and severe hypertension); however, these patients represent only about onethird of all people diagnosed with hypertension, while the other two-thirds have mild hypertension.

Screening for hypertension is controversial. One goodquality randomized controlled trial of 140.642 people of blood pressure screening program, [4] suggested that there were 3 fewer annual hospital admissions for cardiovascular disease per 1000 persons in the intervention group. On the other hand, also in the intervention group, new antihypertensive prescription was $10 \%$ higher.

From a clinical epidemiology perspective, the thresholds defined in guidelines for hypertension have also been controvertial [5-7]. Due to the nature of the relationship between blood pressure and cardiovascular disease, there is an uncertainty range in which the threshold could be set [8]. Gudelines have evolved from defining hypertension with a few measures to including more specific diagnostic tools, such as home blood pressure monitoring for longer periods of time [9]. However, guidelines have tended to lower hypertension definitions [10], besides the lack of evidence to do so [11].

Best available evidence shows that pharmacological treatment for mild hypertension has not been established $[12,13]$. A Cochrane review on pharmacotherapy for mild hypertension concludes that antihypertensive drugs used in the treatment of adults with mild hypertension have not been shown to reduce mortality or morbidity in randomized controlled trials, while $9 \%$ of patients discontinued treatment due to adverse effects. More recently, a longitudinal cohort found no evidence to support the

Table 1 Hypertesion Classification in Brazil

\begin{tabular}{lll}
\hline Classification & Systolic $(\mathrm{mmHg})$ & Diastolic $(\mathrm{mmHg})$ \\
\hline Normal & $<120$ & $<80$ \\
Pre-hypertension & $121-139$ & $81-89$ \\
Stage 1 hypertension (mild) & $140-159$ & $90-99$ \\
Stage 2 hypertension (moderate) & $160-179$ & $100-109$ \\
Stage 3 hypertension (severe) & $>179$ & $>109$ \\
\hline
\end{tabular}

initiation of treatment in patients with mild hypertension. This cohort lasted from 1998 til 2015 and included 19.143 adults who had mild hypertension without comorbidities (low-risk mild hypertension) and no previous treatment.

In addition, the side effects of anti-hypertensive treatment for cardiovascular disease have no relationship with the severity of the baseline condition and are equally distributed on the continuum of hypertension. This equal distribution means that those individuals with mild hypertension have the same risk of unintentional harm when compared with those with moderate to severe hypertension [14].

Moreover, evidence suggests that during the diagnosis process, people are harmed in various other ways besides the typical side effects of medication. This harm can be conceptualised as the psychosocial consequences of being labelled with a diagnosis.

Few qualitative studies have been conducted among people with hypertension to assess aspects related to psychosocial consequences $[15,16]$. One study describes the interviews with 27 patients with hypertension who referred to hospitals. They experienced many physical, psychological, social, familial and spiritual problems which were associated with hypertension. Another study interviewed 6 hospitalized patients undergoing clinical investigation related to hypertension, who described the impact of the diagnosis in their daily lives. However, these two studies did not address people with mild hypertension without comorbidities in a community setting for the psychosocial consequences of labelling hypertension.

One Danish study included people with mild hypertension in a primary healthcare setting and concluded that: the diagnosis of hypertension is a biographical disruption and impacts on daily life and patients' adaptation to hypertension combines biographical and bodily experiences [17].

Recently, we conducted a qualitative study assessing the psychosocial consequences of labelling people in Brazil with mild hypertension. We found that the diagnosis of mild hypertension is a significant event that affects daily life, and most of the impact is regarded by patients as having negative psychosocial consequences or causing harm [18].

These psychosocial consequences have also been the subject of quantitative interventional studies from a medical perspective. The bulk of evidence points to poorer interpersonal relationships, greater absenteeism and increased healthcare service use, among others, as consequences of being labelled as hypertensive [19-25].

Other studies have used instruments designed to assess health-related quality of life and psychological distress, mainly using the SF-12 and SF-36 with people with hypertension [26]. However, these generic instruments potentially lack content validity: they address topics that are irrelevant to individuals with hypertension and do not comprehensively address all relevant topics [27-29]. 
Furthermore, psychosocial aspects of life are typically assessed via patient-oriented perspectives. Specific questionnaires have been developed and used to assess quality of life in people with hypertension (e.g. CHAL and MINICHAL). However, these questionnaires were not developed from the patients' perspective; thus, they potentially also lack content validity [30-33].

More recently, with patient-centred medicine helping to balancing the doctor and the patient perspectives, Patient Reported Outcome Measures (PROMs) emerged [34] with items used to measure psychosocial attributes oriented by the patients' perspectives [35]. These perspectives can be assessed qualitatively to generate items, which are later tested for content validity to create a draft questionnaire that can then be investigated for its psychometric properties [36].

An example of this type of questionnaire is the Consequences of Screening (COS) questionnaire [37-40]. COS is a family of questionnaires addressing various screening scenarios for life-threatening diseases, which is not the case of hypertension. However, it has been shown in qualitative studies that in spite of these differences in severity, living with life-threatening diseases share similarities to living with hypertension [41]. The first questionnaire of this series was developed to capture the psychosocial consequences of abnormal and false-positive screening mammography for breast cancer and was named the COS-BC [37]. Later, additional versions were developed to address other screening scenarios for life-threatening, non-communicable diseases, including lung cancer, abdominal aortic aneurism and cervical cancer [38-40]. The COS questionnaires were developed in Danish and have a two-part common core questionnaire, in which the first part measures the negative psychosocial consequences at any time during the screening process, while the second part assesses changes in the longterm psychosocial consequences of screening after a final diagnosis.

We hypothesised that if people diagnosed with mild hypertension regarded this diagnosis as a life-threatening disease, then at least the core items and dimensions and possibly some of the condition-specific COS items would be relevant to people labelled with mild hypertension. Therefore, the overall purpose of this study was to develop a pool of items for a condition-specific draft questionnaire with high content validity to measure the psychosocial consequences of being diagnosed with mild hypertension. The steps to reach this aim were as follows:

1. Conduct a systematic literature search for questionnaires other than $\operatorname{COS}$ that address issues in hypertension and patient-oriented outcomes in terms of the consequences of labelling;

2. Translate and adapt all the COS core items and all the relevant condition-specific items from other COS questionnaires into Brazilian Portuguese;
3. Assess the content relevance and content coverage of the Brazilian Portuguese core and conditionspecific items in patients diagnosed with mild hypertension;

4. Generate new condition-specific domains and items especially relevant for patient diagnosed with mild hypertension if a lack of content coverage of the Brazilian Portuguese core and condition-specific COS items was revealed;

5. Assess all instructions and items for functionality and understandability.

Later, this pool of items will be tested in a survey, and have their psychometric properties assessed. The psychometric results will be published later.

\section{Methods}

To assess face validity, a systematic literature search was conducted in Medline and PsycINFO for articles in English and Portuguese to identify questionnaires used to assess the psychosocial consequences of being diagnosed with hypertension. We used a broad set of search terms related to hypertension (high blood pressure, blood pressure, arterial pressure, hypertension and risk factor); labelling (diagnosis, stereotyping, stigma and awareness) and PROMs (quality of life, patient outcomes, surveys, questionnaires and patient-reported outcome measures). We selected the questionnaires that suited our needs and then cherry picked all items that seemed relevant.

\section{Dual panel}

We translated the all relevant items from the selected questionnaires into Brazilian Portuguese using the dualpanel method [42].

\section{Bilingual panel}

First, in São Paulo, we conducted a bilingual panel including two researchers and four people who were bilingual (fluent in Danish and with mother tongue in Brazilian Portuguese). The panel members were asked to translate all instructions and items from Danish into Brazilian Portuguese. If there were divergences in the translations, they were asked to discuss and find a consensual translation. If the panel members could not reach consensus they were allowed to generate two or more versions and leave it up to the next panel to decide which translation was most close to lay Brazilian Portuguese language.

\section{Lay panel}

Second, the lay panel included people living in São Paulo and who had no knowledge of the Danish language. The members of the lay panel were five community healthcare workers $(\mathrm{CHW})$. In addition, one of the bilingual experts helped JB during the lay panel. He translated 
discussions and questions from Portuguese into Danish and vice-versa. The translated items were read together with the group, and we asked if the versions produced by the bilingual panel were expressed in easily understandable lay language. After this session, a draft of the Consequences of Hypertension $(\mathrm{COH})$ questionnaire in Brazilian Portuguese was drafted.

\section{Interviews}

The recruitment of informants has previously been described in details [18]. In short, we recruited the informants for interviews ad hoc from public primary healthcare services in São Paulo (known as Unidade Básica de SaúdeUBS), social media and social networks. They were strategically recruited to obtain a wide range of experiences and ages, times from diagnosis, education levels and ethnic groups and both sexes. People recruited from the UBS were identified from the list of people diagnosed with hypertension. People recruited via social media responded to an invitation posted on Facebook. Two of the informants were recruited via the researchers' own social network. A telephone interview was conducted prior to the face-to-face interviews to assess inclusion and exclusion criteria.

Inclusion criteria: Raised in Brazil, confirmed diagnosis of mild hypertension by a physician, prescribed antihypertensive treatment and with no other chronic or disabling conditions.

All interviews were digitally audio recorded and transcribed verbatim.

\section{In-depth individual interviews}

Eleven 1-2 h semi-structured, individual in-depth interviews were conducted between October 2016 and March 2017 at a location the informants found least inconvenient (Table 4 in Appendix).

After the in-depth interviews, the informants completed the draft $\mathrm{COH}$ during a think-aloud session [43]. They were asked to formulate opinions on the instructions, on the items and on the layout of the questionnaire.

Later, we read and discussed the content of the interviews; if lack of content coverage was identified, we formulated new items that reflected informants' verbatim expressions (whenever possible), categorised the items into previous domains and suggested new domains when new items did not fit into the previous domains. These new domains and items were then added to the $\mathrm{COH}$ for the next steps.

\section{Focus groups}

Next, we conducted four 2-h focus-group interviews in an easy-to-access location (Table 4 in Appendix). The informants included in these interviews were grouped strategically with similar characteristics regarding sex and education level.

The focus-group interviews consisted of two parts: first, we led an open-ended discussion for about $30 \mathrm{~min}$, and for the next 90 min, we discussed the draft $\mathrm{COH}$ questionnaire. All the items were tested, but we first focused on the newly generated items. We asked the group if the items were understandable, represented experiences that they might have had (content relevance) and if there were any domains or items missing or irrelevant (content coverage).

\section{Structured interviews}

Finally, we conducted four 60 -min structured individual interviews. (Table 4 in Appendix) The informants were given a list of all items and asked to elaborate on all of the new condition-specific items and to categorise them into pre-determined domains.

Given that recently elaborated items were tested, the informants were told they could be categorised into one of the existing domains, or if necessary, a new domain could be suggested. Similar suggestions on an item were considered powerful enough to categorize that item or to lead to the creation of a new domain, while items without similar suggestions were left for later discussion among the authors.

\section{Results}

\section{Literature search}

No condition-specific PROM on the consequences of labelling people with hypertension was identified. Therefore, the COS questionnaires were chosen as the only relevant source of items.

We selected 76 items (55 items from part I and 21 from part II) from the 4 COS questionnaires; Half of which ( 26 from part I and 12 from part II) are present in all COS questionnaires and compose the core items. The other half ( 29 from part I and 9 from part II) is present in $\mathrm{COS}$ as disease specific items.

A total of 69 items out of these 76 items were representative of 17 different domains: 12 in part I and 5 in part II, while 7 items were regarded as single items: 2 in part I and 5 in part II. Table 2 lists all items, with their respective Brazilian Portuguese wordings, domains, parts, positions, origins, meaning in English or Danish and response categories. Figure 1 describes all the methodological steps and results of the present study.

\section{Dual panel}

Three 76 core items generated more than 1 version in Brazilian Portuguese, resulting in a total of 80 items.

\section{Bilingual panel}

All original Danish items, except three from part I, did reach consensual Brazilian translation. These three items were given more than one Brazilian version: Items 16, 93 and 94 were three Brazilian items representing different translated and adaptated versions of the original Danish item 16 ('I felt bothered'); items 19 and 20 are two versions of original Danish item 19 ('I felt paralyzed'); and items 3 and 4 are also 
Table 2 All items in Brazilian Portuguese and the ad hoc translation. Items in part II are sentences completed with the response categories provided. For example: item 97, which is 'my joy of living became...' can be completed with 'the same as before'. Item 95 ('do you have high blood pressure? yes/no') is not included in the list. This item will be used to determine those who are required to complete part II

\begin{tabular}{|c|c|c|c|c|c|}
\hline Part & Position & Brazilian Portuguese version & $\begin{array}{l}\text { Questionnaire } \\
\text { of origin }\end{array}$ & Domain & ad hoc English translation \\
\hline । & 1 & Me senti preocupado & core & $\begin{array}{l}\text { Sense of } \\
\text { dejection }\end{array}$ & I felt worried \\
\hline । & 2 & Me senti preocupado com meu futuro & core & Anxiety & I felt worried about my future \\
\hline । & 3 & Me senti amedrontado & core & Anxiety & I felt frightened \\
\hline । & 4 & Me senti com medo & core & Anxiety & I felt scared \\
\hline । & 5 & Me senti irritado & core & Behaviour & I felt annoyed \\
\hline । & 6 & Me senti mais quieto do que o normal & core & Behaviour & I felt quieter than usual \\
\hline । & 7 & Dormi mal à noite & core & Sleep & I slept badly at night \\
\hline । & 8 & $\begin{array}{l}\text { Fuji dos meus pensamentos me ocupando com } \\
\text { tarefas práticas do dia-a-dia }\end{array}$ & core & Single Items & $\begin{array}{l}\text { I ran away from my thoughts, busy with day- } \\
\text { to-day practical tasks }\end{array}$ \\
\hline 1 & 9 & Me senti com dificuldade de me concentrar & core & Behaviour & I felt hard to concentrate \\
\hline । & 10 & $\begin{array}{l}\text { Me senti com a sensação de que o tempo não } \\
\text { passava }\end{array}$ & core & $\begin{array}{l}\text { Sense of } \\
\text { dejection }\end{array}$ & I felt that time was not passing \\
\hline । & 11 & Tive mudanças em meu apetite & core & Behaviour & I had changes in my appetite \\
\hline । & 12 & Me senti triste & core & $\begin{array}{l}\text { Sense of } \\
\text { dejection }\end{array}$ & I felt sad \\
\hline । & 13 & Me senti emotionalmente fora do meu normal & core & Anxiety & I felt emotionally out of my normal \\
\hline । & 14 & Me senti inquieto & core & Anxiety & I felt restless \\
\hline । & 15 & Me senti nervoso & core & Anxiety & I felt nervous \\
\hline । & 16 & Me senti ansioso & core & Anxiety & I felt anxious \\
\hline 1 & 17 & Tive dificuldade de pegar no sono & core & Sleep & I had difficulty falling asleep \\
\hline । & 18 & Me senti mais fechado & core & Behaviour & I felt introvert \\
\hline 1 & 19 & Me senti sem iniciativa & core & $\begin{array}{l}\text { Sense of } \\
\text { dejection }\end{array}$ & I felt without initiative \\
\hline 1 & 20 & Me senti sem vontade & core & $\begin{array}{l}\text { Sense of } \\
\text { dejection }\end{array}$ & I felt unwilling \\
\hline 1 & 21 & Me senti deprimido & core & $\begin{array}{l}\text { Sense of } \\
\text { dejection }\end{array}$ & I felt depressed \\
\hline 1 & 22 & $\begin{array}{l}\text { Tive dificuldades em realizar meu trabalho e } \\
\text { outras tarefas semelhantes }\end{array}$ & core & Behaviour & $\begin{array}{l}\text { I had difficulty doing my job and other similar } \\
\text { tasks }\end{array}$ \\
\hline । & 23 & Acordei cedo demais & core & Sleep & I woke up too early \\
\hline । & 24 & Tive dificuldades em realizar tarefas de casa & core & Behaviour & I had difficulty doing domestic work \\
\hline । & 25 & Me senti a ponto de entrar em pânico & core & Anxiety & I felt about to panic \\
\hline । & 26 & Passei a maior parte do tempo acordado & core & Sleep & I spent most of the time awake \\
\hline । & 27 & Tive menos desejo sexual & core & Sexual & I had less sexual desire \\
\hline । & 28 & Dias faltados no trabalho & core & Single Items & Days missed at work \\
\hline 1 & 29 & Me senti em estado de choque & $\begin{array}{l}\text { disease } \\
\text { specific }\end{array}$ & Anxiety & I felt in shock \\
\hline । & 30 & $\begin{array}{l}\text { Fiquei com medo da pressão alta o tempo todo } \\
\text { na cabeça }\end{array}$ & new & $\begin{array}{l}\text { Blood pressure } \\
\text { related }\end{array}$ & $\begin{array}{l}\text { I had the fear of high blood pressure all of the } \\
\text { time in the head }\end{array}$ \\
\hline । & 31 & Me senti inseguro & $\begin{array}{l}\text { disease } \\
\text { specific }\end{array}$ & Introvert & I felt insecure \\
\hline । & 32 & Me senti com pena de mim mesmo & $\begin{array}{l}\text { disease } \\
\text { specific }\end{array}$ & Introvert & I felt sorry for myself \\
\hline । & 33 & Me senti em uma situação desesperadora & $\begin{array}{l}\text { disease } \\
\text { specific }\end{array}$ & Introvert & I felt in a desperate situation \\
\hline
\end{tabular}


Table 2 All items in Brazilian Portuguese and the ad hoc translation. Items in part II are sentences completed with the response categories provided. For example: item 97, which is 'my joy of living became...' can be completed with 'the same as before'. Item 95 ('do you have high blood pressure? yes/no') is not included in the list. This item will be used to determine those who are required to complete part II (Continued)

\begin{tabular}{|c|c|c|c|c|c|}
\hline Part & Position & Brazilian Portuguese version & $\begin{array}{l}\text { Questionnaire } \\
\text { of origin }\end{array}$ & Domain & ad hoc English translation \\
\hline I & 34 & Fiquei com humor muito variável & $\begin{array}{l}\text { disease } \\
\text { specific }\end{array}$ & Introvert & I was in a very variable mood \\
\hline । & 35 & Me senti mais cansado do que de costume & $\begin{array}{l}\text { disease } \\
\text { specific }\end{array}$ & Single Items & I felt more tired than usual \\
\hline । & 36 & Guardei meus pensamentos só pra mim & $\begin{array}{l}\text { disease } \\
\text { specific }\end{array}$ & Single Items & I kept my thoughts just for myself \\
\hline । & 37 & Me senti doente & $\begin{array}{l}\text { disease } \\
\text { specific }\end{array}$ & Body Perception & I felt sick \\
\hline । & 38 & $\begin{array}{l}\text { Tive a sensação de que havia algo errado com } \\
\text { meu corpo }\end{array}$ & $\begin{array}{l}\text { disease } \\
\text { specific }\end{array}$ & Body Perception & $\begin{array}{l}\text { I had a feeling something was wrong with my } \\
\text { body }\end{array}$ \\
\hline । & 39 & Me senti fora de controle & $\begin{array}{l}\text { disease } \\
\text { specific }\end{array}$ & $\begin{array}{l}\text { Fear and } \\
\text { Powerlessness }\end{array}$ & I felt out of control \\
\hline । & 40 & Me senti com o corpo frágil & $\begin{array}{l}\text { disease } \\
\text { specific }\end{array}$ & $\begin{array}{l}\text { Fear and } \\
\text { Powerlessness }\end{array}$ & I felt my body fragile \\
\hline । & 41 & Senti que a idade chegou & $\begin{array}{l}\text { disease } \\
\text { specific }\end{array}$ & Perception of age & I felt that old age has come \\
\hline । & 42 & $\begin{array}{l}\text { Me senti como se meu corpo fosse uma } \\
\text { máquina que não funciona }\end{array}$ & $\begin{array}{l}\text { disease } \\
\text { specific }\end{array}$ & Body Perception & I felt like my body was a non-working machine \\
\hline । & 43 & Me senti azedo & $\begin{array}{l}\text { disease } \\
\text { specific }\end{array}$ & Emotional & I felt sour \\
\hline । & 44 & Me senti zangado & $\begin{array}{l}\text { disease } \\
\text { specific }\end{array}$ & Emotional & I felt angry \\
\hline । & 45 & Me senti como se estivesse no vazio & $\begin{array}{l}\text { disease } \\
\text { specific }\end{array}$ & Single Items & I felt like I was in the void \\
\hline । & 46 & $\begin{array}{l}\text { Me senti como um estranho em meu próprio } \\
\text { corpo }\end{array}$ & $\begin{array}{l}\text { disease } \\
\text { specific }\end{array}$ & Body Perception & I felt like a stranger in my own body \\
\hline । & 47 & Me senti mais velho do que sou & $\begin{array}{l}\text { disease } \\
\text { specific }\end{array}$ & Perception of age & I felt older than I am \\
\hline । & 48 & Me senti sem forças & $\begin{array}{l}\text { disease } \\
\text { specific }\end{array}$ & $\begin{array}{l}\text { Fear and } \\
\text { Powerlessness }\end{array}$ & I felt strengthless \\
\hline । & 49 & Chorei mais do que de costume & $\begin{array}{l}\text { disease } \\
\text { specific }\end{array}$ & Emotional & I cried more than usual \\
\hline । & 50 & Me senti sem sorte & $\begin{array}{l}\text { disease } \\
\text { specific }\end{array}$ & $\begin{array}{l}\text { Fear and } \\
\text { Powerlessness }\end{array}$ & I felt unlucky \\
\hline । & 51 & Me senti vulnerável & $\begin{array}{l}\text { disease } \\
\text { specific }\end{array}$ & $\begin{array}{l}\text { Fear and } \\
\text { Powerlessness }\end{array}$ & I felt vulnerable \\
\hline । & 52 & Me senti fragilizado & $\begin{array}{l}\text { disease } \\
\text { specific }\end{array}$ & Single Items & I felt weak \\
\hline । & 53 & $\begin{array}{l}\text { Me senti como se qualquer coisa pudesse me } \\
\text { afetar }\end{array}$ & $\begin{array}{l}\text { disease } \\
\text { specific }\end{array}$ & Body Perception & I felt like anything could affect me \\
\hline । & 54 & Mudei meus hábitos de atividade física & $\begin{array}{l}\text { disease } \\
\text { specific }\end{array}$ & Lifestyle & I changed my exercising habits \\
\hline । & 55 & Pensei na morte & $\begin{array}{l}\text { disease } \\
\text { specific }\end{array}$ & Single Items & I thought about death \\
\hline । & 56 & Mudei meus hábitos alimentares & $\begin{array}{l}\text { disease } \\
\text { specific }\end{array}$ & Lifestyle & I changed my eating habits \\
\hline । & 57 & $\begin{array}{l}\text { Pensei que seria melhor se não soubesse que } \\
\text { tenho pressão alta }\end{array}$ & new & $\begin{array}{l}\text { Blood pressure } \\
\text { related }\end{array}$ & $\begin{array}{l}\text { I thought it would be better if I didn't know I } \\
\text { have high blood pressure }\end{array}$ \\
\hline । & 58 & Tive medo de fazer esforço físico & $\begin{array}{l}\text { disease } \\
\text { specific }\end{array}$ & $\begin{array}{l}\text { Fear and } \\
\text { Powerlessness }\end{array}$ & I was afraid of doing exercises \\
\hline
\end{tabular}


Table 2 All items in Brazilian Portuguese and the ad hoc translation. Items in part II are sentences completed with the response categories provided. For example: item 97, which is 'my joy of living became...' can be completed with 'the same as before'. Item 95 ('do you have high blood pressure? yes/no') is not included in the list. This item will be used to determine those who are required to complete part II (Continued)

\begin{tabular}{|c|c|c|c|c|c|}
\hline Part & Position & Brazilian Portuguese version & $\begin{array}{l}\text { Questionnaire } \\
\text { of origin }\end{array}$ & Domain & ad hoc English translation \\
\hline । & 59 & Me senti insatisfeito com minha vida sexual & $\begin{array}{l}\text { disease } \\
\text { specific }\end{array}$ & Sexual & I felt dissatisfied with my sex life \\
\hline । & 60 & Pensei na minha fé & new & Single Items & I thought of my faith \\
\hline । & 61 & Me senti impaciente & new & Anxiety & I felt impatient \\
\hline । & 62 & Me senti culpado & new & $\begin{array}{l}\text { Sense of } \\
\text { dejection }\end{array}$ & I felt guilty \\
\hline । & 63 & Me senti desequilibrado & new & Emotional & I felt unbalanced \\
\hline । & 64 & Senti que não tenho saúde & new & Body Perception & I felt that I am not healthy \\
\hline 1 & 65 & Me senti em dúvida & new & $\begin{array}{l}\text { Results of } \\
\text { diagnosis }\end{array}$ & I felt in doubt \\
\hline । & 66 & Me senti sem saber o que esperar & new & $\begin{array}{l}\text { Fear and } \\
\text { Powerlessness }\end{array}$ & I didn't know what to expect \\
\hline । & 67 & Me senti desmotivado & new & $\begin{array}{l}\text { Sense of } \\
\text { dejection }\end{array}$ & I felt unmotivated \\
\hline । & 68 & Me senti desestimulado & new & $\begin{array}{l}\text { Sense of } \\
\text { dejection }\end{array}$ & I felt discouraged \\
\hline । & 69 & Me senti fraco & new & Body Perception & I felt weak \\
\hline । & 70 & Me senti frustrado & new & $\begin{array}{l}\text { Sense of } \\
\text { dejection }\end{array}$ & I felt frustrated \\
\hline । & 71 & Me senti indiferente & new & $\begin{array}{l}\text { Sense of } \\
\text { dejection }\end{array}$ & I felt indifferent \\
\hline I & 72 & Me senti sendo julgado & new & Social Relations & I felt being judged \\
\hline । & 73 & Me senti com pavor & new & $\begin{array}{l}\text { Fear and } \\
\text { Powerlessness }\end{array}$ & I felt terrified \\
\hline । & 74 & Me senti preso & new & Emotional & I felt trapped \\
\hline । & 75 & $\begin{array}{l}\text { Me senti sendo forçado a fazer coisas que não } \\
\text { quero }\end{array}$ & new & Single Items & I felt being forced to do things I don't want \\
\hline l & 76 & Me senti orgulhoso & new & Emotional & I felt proud \\
\hline । & 77 & Me senti apreensivo & new & $\begin{array}{l}\text { Fear and } \\
\text { Powerlessness }\end{array}$ & I felt apprehensive \\
\hline । & 78 & Me senti com raiva & new & Emotional & I felt angry \\
\hline । & 79 & Me senti impotente & new & $\begin{array}{l}\text { Fear and } \\
\text { Powerlessness }\end{array}$ & I felt helpless \\
\hline । & 80 & Me senti surpreso & new & $\begin{array}{l}\text { Results of } \\
\text { diagnosis }\end{array}$ & I felt surprised \\
\hline । & 81 & Me senti tranquilo & new & Single Items & I felt calm \\
\hline । & 82 & Me senti chateado & new & $\begin{array}{l}\text { Sense of } \\
\text { dejection }\end{array}$ & I felt upset \\
\hline । & 83 & Me senti envergonhado & new & Emotional & I felt ashamed \\
\hline । & 84 & Me senti controlado pelos outros & new & Social Relations & I felt controlled by others \\
\hline । & 85 & Me senti apoiado & new & Social Relations & I felt supported \\
\hline । & 86 & Me senti excluído & new & Social Relations & I felt excluded \\
\hline । & 87 & Me senti cuidado & new & Social Relations & I felt being cared for \\
\hline । & 88 & Me senti diferente & new & Social Relations & I felt different \\
\hline । & 89 & Me senti importante & new & Social Relations & I felt important \\
\hline । & 90 & Tive sintomas de pressão alta & new & Blood pressure & I had symptoms of high blood pressure \\
\hline
\end{tabular}


Table 2 All items in Brazilian Portuguese and the ad hoc translation. Items in part II are sentences completed with the response categories provided. For example: item 97, which is 'my joy of living became...' can be completed with 'the same as before'. Item 95 ('do you have high blood pressure? yes/no') is not included in the list. This item will be used to determine those who are required to complete part II (Continued)

\begin{tabular}{|c|c|c|c|c|c|}
\hline Part & Position & Brazilian Portuguese version & $\begin{array}{l}\text { Questionnaire } \\
\text { of origin }\end{array}$ & Domain & ad hoc English translation \\
\hline & & & & related & \\
\hline I & 91 & $\begin{array}{l}\text { Me senti culpado por não cuidar de mim } \\
\text { mesmo como deveria }\end{array}$ & new & $\begin{array}{l}\text { Sense of } \\
\text { dejection }\end{array}$ & $\begin{array}{l}\text { I felt guilty for not taking care of myself } \\
\text { as I should }\end{array}$ \\
\hline । & 92 & Me senti assustado & new & $\begin{array}{l}\text { Fear and } \\
\text { Powerlessness }\end{array}$ & I felt scared \\
\hline 1 & 93 & Me senti agitado & core & Anxiety & I felt agitated \\
\hline । & 94 & Me senti incomodado & core & $\begin{array}{l}\text { Sense of } \\
\text { dejection }\end{array}$ & I felt bothered \\
\hline$\|$ & 96 & eu fiquei pensando na vida & core & Existential values & I kept thinking about life \\
\hline$\|$ & 97 & minha alegria de viver ficou & core & Existential values & my joy of living became... \\
\hline$\|$ & 98 & me senti tranquilo & core & Relaxed/Calm & I felt calm \\
\hline$\|$ & 99 & a minha relação com a minha família ficou & core & Personal Relations & my relationship with my family became... \\
\hline$\|$ & 100 & a minha relação com meus amigos ficou & core & Personal Relations & my relationship with my friends became... \\
\hline$\|$ & 101 & a minha relação com outras pessoas ficou & core & Personal Relations & my relationship with other people became... \\
\hline ॥ & 102 & me senti calmo & core & Relaxed/Calm & I felt calm \\
\hline ॥ & 103 & a minha visão do futuro ficou & core & Existential values & my vision of the future became... \\
\hline$\|$ & 104 & a minha sensação de bem-estar ficou & core & Existential values & my sense of well-being became... \\
\hline$\|$ & 105 & a minha percepção sobre a vida ficou & core & Existential values & my perception of life became... \\
\hline$\|$ & 106 & o valor que dou a vida ficou & core & Existential values & the value I give in life became... \\
\hline$\|$ & 107 & a minha energia ficou & $\begin{array}{l}\text { disease } \\
\text { specific }\end{array}$ & Impulsive & my energy became... \\
\hline$\|$ & 108 & $\begin{array}{l}\text { meu sentimento de responsabilidade pela } \\
\text { minha família ficou }\end{array}$ & $\begin{array}{l}\text { disease } \\
\text { specific }\end{array}$ & Empathy & $\begin{array}{l}\text { my sense of responsibility for my family } \\
\text { became... }\end{array}$ \\
\hline$\|$ & 109 & tenho aproveitado a vida & $\begin{array}{l}\text { disease } \\
\text { specific }\end{array}$ & Impulsive & I have enjoyed life \\
\hline$\|$ & 110 & me sinto aliviado & core & Relaxed/Calm & I feel relieved \\
\hline$\|$ & 111 & $\begin{array}{l}\text { minha compreensão dos problemas alheios } \\
\text { ficou }\end{array}$ & $\begin{array}{l}\text { disease } \\
\text { specific }\end{array}$ & Empathy & $\begin{array}{l}\text { my understanding of other people's problems } \\
\text { became... }\end{array}$ \\
\hline$\|$ & 112 & me sinto impulsivo & $\begin{array}{l}\text { disease } \\
\text { specific }\end{array}$ & Impulsive & I feel impulsive \\
\hline$\|$ & 113 & $\begin{array}{l}\text { a minha capacidade de ouvir problemas alheios } \\
\text { ficou }\end{array}$ & $\begin{array}{l}\text { disease } \\
\text { specific }\end{array}$ & Empathy & $\begin{array}{l}\text { my ability to hear other people's problems } \\
\text { became... }\end{array}$ \\
\hline$\|$ & 114 & $\begin{array}{l}\text { a minha vontade de me envolver com algo } \\
\text { novo ficou }\end{array}$ & $\begin{array}{l}\text { disease } \\
\text { specific }\end{array}$ & Impulsive & $\begin{array}{l}\text { my desire to get involved with something new } \\
\text { became... }\end{array}$ \\
\hline$\|$ & 115 & $\begin{array}{l}\text { a minha vontade de me envolver com algo } \\
\text { arriscado ficou }\end{array}$ & $\begin{array}{l}\text { disease } \\
\text { specific }\end{array}$ & Impulsive & $\begin{array}{l}\text { my desire to get involved with something risky } \\
\text { got... }\end{array}$ \\
\hline$\|$ & 116 & tenho feito coisas que utrapassam meus limites & $\begin{array}{l}\text { disease } \\
\text { specific }\end{array}$ & Impulsive & I've been doing things that push my limits \\
\hline$\|$ & 117 & frequento consultas médicas & new & Patient Role & I go to doctor's appointments \\
\hline$\|$ & 118 & faço exames & new & Patient Role & I take exams \\
\hline$\|$ & 119 & me sinto fazendo mal para mim mesmo & new & Patient Role & I feel bad for myself \\
\hline$\|$ & 120 & $\begin{array}{l}\text { me sinto com dificuldades em seguir } \\
\text { orientações médicas }\end{array}$ & new & Patient Role & I have difficulty following medical advice \\
\hline$\|$ & 121 & me sinto cuidando de mim mesmo & new & Patient Role & I feel taking care of myself \\
\hline$\|$ & 122 & tomo medicamentos & new & Patient Role & I take medicines \\
\hline$\|$ & 123 & me sinto dependente de remédios & new & Patient Role & I feel dependent on medicines \\
\hline
\end{tabular}


Table 2 All items in Brazilian Portuguese and the ad hoc translation. Items in part II are sentences completed with the response categories provided. For example: item 97, which is 'my joy of living became...' can be completed with 'the same as before'. Item 95 ('do you have high blood pressure? yes/no') is not included in the list. This item will be used to determine those who are required to complete part II (Continued)

\begin{tabular}{|c|c|c|c|c|c|}
\hline Part & Position & Brazilian Portuguese version & $\begin{array}{l}\text { Questionnaire } \\
\text { of origin }\end{array}$ & Domain & ad hoc English translation \\
\hline$\|$ & 124 & me sinto confiante em orientações médicas & new & Patient Role & I feel confident in medical advice \\
\hline$\|$ & 125 & me sinto como se não fosse mais normal & new & Existential values & I feel like I'm not normal anymore \\
\hline$\|$ & 126 & me sinto como se não fosse mais o mesmo & new & Existential values & I feel like I'm not the same anymore \\
\hline$\|$ & 127 & $\begin{array}{l}\text { me sinto preocupado com sintomas de } \\
\text { pressão alta }\end{array}$ & new & $\begin{array}{l}\text { Preoccupation } \\
\text { with health }\end{array}$ & $\begin{array}{l}\text { I feel worried about symptoms of high } \\
\text { blood pressure }\end{array}$ \\
\hline$\|$ & 128 & $\begin{array}{l}\text { me sinto preocupado com meus hábitos e } \\
\text { estilo de vida }\end{array}$ & new & $\begin{array}{l}\text { Preoccupation } \\
\text { with health }\end{array}$ & I feel worried about my habits and lifestyle \\
\hline$\|$ & 129 & me sinto preocupado com os tratamentos & new & $\begin{array}{l}\text { Preoccupation } \\
\text { with health }\end{array}$ & I feel worried about the treatments \\
\hline$\|$ & 130 & meu desempenho no trabalho ficou & new & Single Items & my work performance became... \\
\hline$\|$ & 131 & minha prática sexual ficou & new & Single Items & my sexual practice became... \\
\hline$\|$ & 132 & $\begin{array}{l}\text { minha ansiedade com relação a pressão alta } \\
\text { ficou }\end{array}$ & new & $\begin{array}{l}\text { Hypertension } \\
\text { related }\end{array}$ & my anxiety about high blood pressure got \\
\hline$\|$ & 133 & penso que eu não tenho pressão alta & new & $\begin{array}{l}\text { Hypertension } \\
\text { related }\end{array}$ & I think I don't have high blood pressure \\
\hline
\end{tabular}

two Brazilian versions of the original Danish item 3 ('I felt scared'). Therefore, after conducting this panel, the 76 original Danish items became 80 Brazilian items.

\section{Lay panel}

The group confirmed the instructions' and items' translations as lay language and understandable. They were unable to select one item out of the versions for items 3, 16 and 19 , and therefore all were kept, confirming all 80 items. One sensitive suggestion was confirmed during this part, which was related to the inversion of the pronominal preposition in Brazilian Portuguese. Although this represents a grammatically incorrect form of the sentences, it is directly related to the way Brazilian people speak. All the sentences were then rewritten from 'Senti-me...' to 'Me senti ...'

\section{Interviews}

Altogether we included in all three kinds of interviews 27 informants of both sexes, aged 21-74 years, being diagnosed with hypertension 1 month to 30 years ago, education level low to high, including illiteracy, and various ethnic groups.

Our informants content-validated the 80 translated items. In total, we generated 52 new items ( 35 for part I and 17 for part II) for 12 domains. Twenty-five of these new were encompassed in 6 new domains. In part I 3 new domains were generated: the 'blood pressurerelated' domain encompassing 3 items, the 'social relations' domain encompassing 7 items and the 'results of diagnosis' domain encompassing 2 items. In part II 3 new domains were also generated: the 'hypertension-related' domain encompassing 2 items, the 'patient role' domain encompassing 8 items and the 'preoccupation with health' domain encompassing 3 items.

\section{In-depth individual interviews}

Instructions Three options of instructions for part I were designed based on our previous experiences with questionnaires. We offered our informants these three options and asked them to elaborate on them:

- How have you been feeling the last month? (Como você se sentiu no ultimo mês?) (Or)

- How have you been feeling the last week? (Como você se sentiu no ultimo semana?) (Or)

- How do you feel nowadays regarding blood pressure? (Como você se sente hoje em dia com relação à pressão?)

The informants suggested that the best way to frame the instruction of part I was the first option: 'how have you been feeling the last month?', and we chose this one for the questionnaire. They suggested that the second option included a too short of a period, while the third was rejected beacause it was too broad.

Complementary part II was opened with the question: Taking everything into account: the diagnosis, the follow-up, the exams, the pills... (Levando tudo em consideração: o diagnostico, o seguimento, os exames, os remédios ...); and part II has items introduced by the sentence: ... after I knew I had high blood pressure ... (... depois de saber que tenho pressão alta ...). No changes were suggested in this part. 


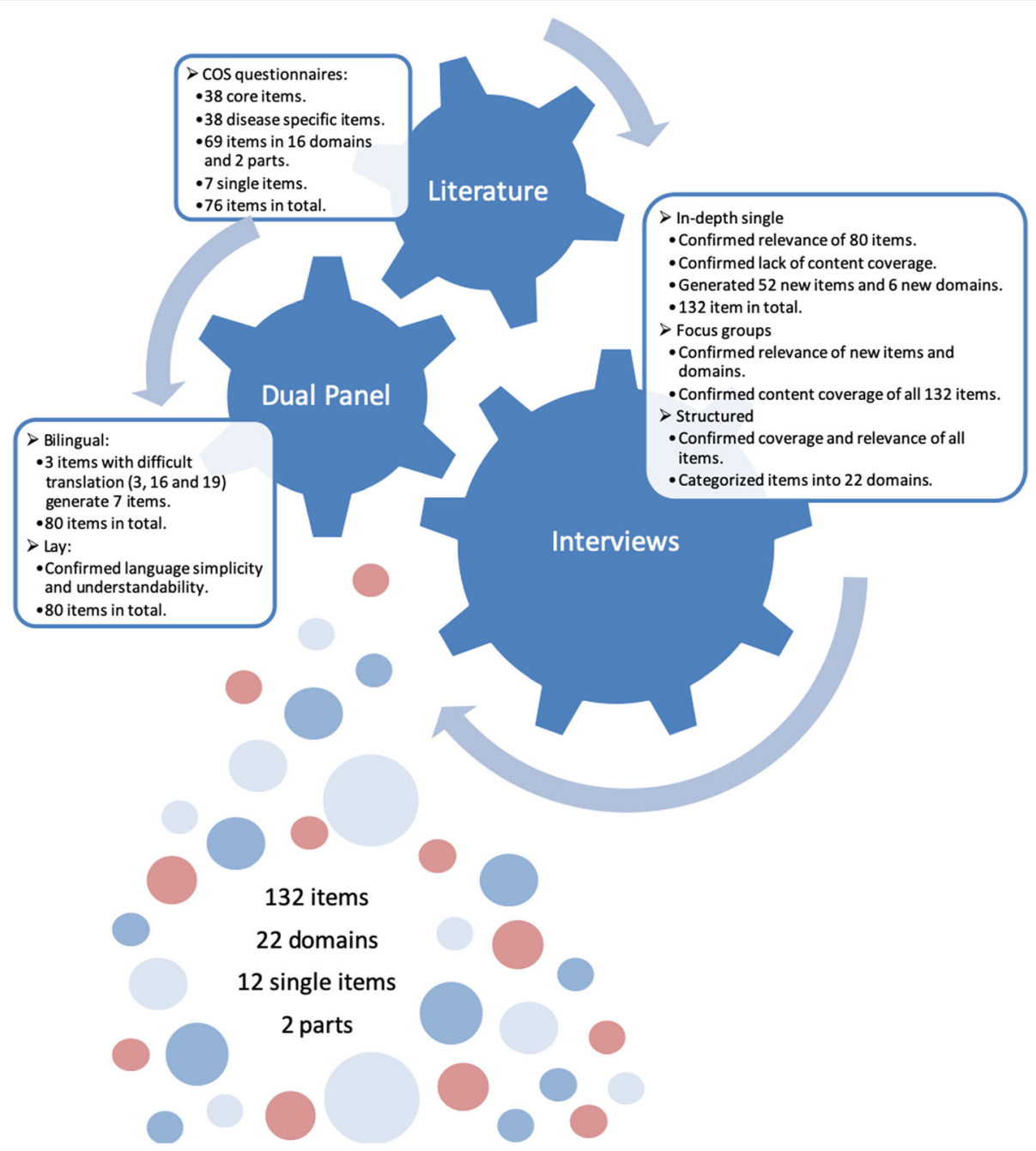

Fig. 1 Material, Methods and Results

Response categories The original COS was developed with polytomous items. Part I had the following possible answers:

- No, not at all/no, not even once (não, nem um pouco/não, nem uma vez)

- Yes, a little/yes, a few times (sim, um pouco/sim, poucas vezes)

- Yes, some/yes, sometimes (sim, não muito/sim, às vezes)

- Yes, a lot/yes, many times (sim, muito/sim, muitas vezes)

A few items had a fifth option: I don't know (não sei), and one item was relevant to counting the number of missing days at work and had the option: $0,1-2,3-4$ or 5 or more; I don't work. These response categories were confirmed to be relevant, comprehensive, understandable and easy to complete.
The same was found for the translation of the original response categories in part 2. All items were polytomous, with the following possible answers:

- A lot less... (muito menos)

- Some less... (um pouco menos)

- The same as before...(o mesmo que antes)

- Some more... (um pouco mais)

- A lot more...(muito mais)

\section{Focus groups}

No new items were developed. The groups confirmed high content validity of the 132 items.

\section{Structured interviews}

Five new items (3 from part I and 2 from part II) could not be categorised by the informants into any of the existing domains and were therefore regarded as single items 
(Table 3). We also asked the informants to allocate the versions of the two original items without a consensual translation to a domain: items 16, 93 and 94 (originally item 16) and items 19 and 20 (originally item 19). Items 16 and 93 were categorised in a different domain ('anxiety') compared to item 94 that stayed in the original domain ('sense of dejection'). Items 19 and 20 were both categorised as belonging to the domain of 'sense of dejection'.

\section{Discussion}

\section{Major findings}

To achieve high content validity of a measure about psychosocial consequences of being diagnosed with mild hypertension we included a total of 132 items divided into 22 domains in 2 questionnaire parts: Part I encompassed 94 items in 14 domains, part II 38 items in 8 domains.

Ten items remained as single items in part I and two remained in part II. Although a single item does not necessarily have a high measurement precision like a scale, it could be wise to keep these items for content coverage because if a single item has high relevance informants might interprete a questionnaire without such single items as having lack of content coverage: they think important questions are missing.

We did not find any previously published PROMs addressing the psychosocial consequences of labelling people with mild hypertension in our literature search. Qualitative studies describe similar experiences in people living with cancer and people living with cardiovascular disease [40]. Moreover, one of the authors has previously developed the COS questionnaires. The use of previously developed items could be a fast way to the development of new scales, saves time and money and is a common practice: the COS itself was based on previously developed items [37]. We selected the COS questionnaires for the following reasons: accessibility to the content, plausible similar psychosocial consequences between false positives and overdiagnosed in a screening context, the diagnosis of a chronic condition and already established psychometric properties of COS (in Danish and Swedish).

Our choice of translation method was based on its prior use in the development of many other diseasespecific measures in up to 30 languages [44]. Recruiting CHWs as informants for the lay panel was found to be a strength since they have a broad social network and a wide range of cultural experiences and are similar to the target of this questionnaire.

We have generated a very large item pool. This seems like a weakness of this study, since a very long questionnaire might have limited use. However, this is one of the strengths of this study, because it provides a broad range of items for every domain. This broad range of items describes different nuances and will provide enough elements for the psychometric analysis of each domain. It is expected that after the psychometric analysis, the item pool will be significantly reduced.

Face validity was confirmed in the interviews; however, numerous new items had to be added to achieve high content validity of the $\mathrm{COH}$. Another strength of our study is the population for the interviews, which included informants with a broad range of sociodemographic characteristics including health professionals. All of them were residents of São Paulo, which might be a limitation. However, many were migrants from other Brazilian regions. Moreover, we conducted a qualitative study on the psychosocial consequences of being labelled with mild hypertension, and achieved data saturation before conducting any of the group interviews, which might indicate that we had achieved high content coverage for most of the psychosocial consequences of being labelled with mild hypertension.

We asked our informants in single interviews to evaluate 80 translated items from the COS. All the items were found to be relevant and were included in the final draft of the questionnaire. This result might indicate that patients living with the diagnosis of mild hypertension share similarities with those experiencing abnormal results in screening for cancer and abdominal aortic aneurism - diseases that are regarded by most lay people as deadly life-threatning diseases with poor prognoses.

The fact that 52 new items and 6 new domains emerged from our qualitative study indicates that the COS were not comprehensive in a context of mild hypertension. Most of the items were derived directly from transcriptions of words or sentences from the informations verbatim expressions. However, a few were generated based on our analyses of the meaning condensation of the interviews [18]. One example is the item on pride. No informant used the word pride to refer to their experiences, but we noted a sense of pride in their statements referring to efforts and achievements in controlling hypertension and complying with medical prescriptions. The wording of this pride item and other items were confirmed in the following focus-group interviews.

The methods described in this article represent a consistent way to achieve high content validity for PROMs. We used three different qualitative methods because each of them had a different focus and complemented each other, which we see as a strength. Furthermore, if we attempted to address all our needs with every informant, the result would be a very tiresome interview. The purpose of the indepth semi-structured individual interviews was to gain insight into the consequences of labelling mild hypertension, to describe the consequences of this diagnosis, and to test the COS for content validity in this setting. These interviews were also part of our qualitative study on the psychosocial consequences of labelling hypertension [18]. After that, the informants were exposed to a draft version 
Table 3 Dimensions and items

\begin{tabular}{|c|c|}
\hline & Number of items \\
\hline Part I & 94 \\
\hline Anxiety & 11 \\
\hline core & 9 \\
\hline disease specific & 1 \\
\hline new & 1 \\
\hline Behaviour & 7 \\
\hline core & 7 \\
\hline Blood pressure related & 3 \\
\hline new & 3 \\
\hline Body Perception & 7 \\
\hline disease specific & 5 \\
\hline new & 2 \\
\hline Emotional & 8 \\
\hline disease specific & 3 \\
\hline new & 5 \\
\hline Fear and Powerlessness & 11 \\
\hline disease specific & 6 \\
\hline new & 5 \\
\hline Introvert & 4 \\
\hline disease specific & 4 \\
\hline Lifestyle & 2 \\
\hline disease specific & 2 \\
\hline Perception of age & 2 \\
\hline disease specific & 2 \\
\hline Results of diagnosis & 2 \\
\hline new & 2 \\
\hline Sense of dejection & 14 \\
\hline core & 7 \\
\hline new & 7 \\
\hline Sexual & 2 \\
\hline core & 1 \\
\hline disease specific & 1 \\
\hline Single Items & 10 \\
\hline core & 2 \\
\hline disease specific & 5 \\
\hline new & 3 \\
\hline Sleep & 4 \\
\hline core & 4 \\
\hline Social Relations & 7 \\
\hline new & 7 \\
\hline Part II & 38 \\
\hline Empathy & 3 \\
\hline disease specific & 3 \\
\hline Existential values & 8 \\
\hline
\end{tabular}

Table 3 Dimensions and items (Continued)

\begin{tabular}{ll}
\hline & Number of items \\
\hline core & 6 \\
new & 2 \\
Hypertension related & 2 \\
new & 2 \\
Impulsive & 6 \\
disease specific & 6 \\
Patient Role & 8 \\
new & 8 \\
Personal Relations & 3 \\
core & 3 \\
Preoccupation with health & 3 \\
new & 3 \\
Relaxed/Calm & 3 \\
core & 3 \\
Single Items & 3 \\
new & 2 \\
Total Geral & 2 \\
\hline
\end{tabular}

of the questionnaire, allowing them to reflect and evaluate the instructions and the items' content validity. A similar method was used with the focus-group interviews, where we only showed the items after the group had the opportunity for open-ended reflection to discuss and debate the psychosocial consequences of being labelled with mild hypertension.

\section{Conclusion}

High content validity was achieved for a conditionspecific questionnaire measuring the psychosocial consequences of being labelled with mild hypertension. This instrument encompassed 132 items divided into 22 domains in 2 parts. Thereby, a draft of the Consequneces of Hypertension questionnaire $(\mathrm{COH})$ was developed.

\section{Implications for research}

All domains will have to be analysed for unidimensionality and invariant measurement using primarily Item Response Theory Rasch models but also Classical Test Theory, to validate the $\mathrm{COH}$ and thereby select the items for the final version of the $\mathrm{COH}$.

The final questionnaire is intended to be used in hypertension research, specially in hypertension screening scenarios, in which the results might bring to light the unintended psychosocial harms of labelling.

\section{Abbreviations}

$\mathrm{CHW}$ : community healthcare workers; $\mathrm{COH}$ : consequences of hypertension questionnaire; COS: consequences of screening questionnaire; 


\section{Appendix}

Table 4 Demographic characteristics and qualitative method fase

\begin{tabular}{|c|c|c|c|c|c|c|c|}
\hline order & Qualitative phase & Sex & Age & Ethnic & Education (years completed) & Time since diagnosis (years) & Local \\
\hline 1 & semi-structured individual & male & 30 & white & 18 & 4 & public place \\
\hline 2 & semi-structured individual & female & 21 & black & 9 & 4 months & home \\
\hline 3 & semi-structured individual & male & 36 & white & 19 & 3 & work \\
\hline 4 & semi-structured individual & female & 35 & mixed & 8 & 1 month & UBS \\
\hline 5 & semi-structured individual & female & 36 & white & 16 & 4 & home \\
\hline 6 & semi-structured individual & male & 46 & white & 11 & 1.5 & UBS \\
\hline 7 & semi-structured individual & female & 44 & white & 20 & 9 & work \\
\hline 8 & semi-structured individual & female & 64 & white & 11 & 7 & home \\
\hline 9 & semi-structured individual & female & 65 & white & 11 & 22 & home \\
\hline 10 & semi-structured individual & female & 64 & mixed & 11 & 15 & home \\
\hline 11 & semi-structured individual & male & 44 & mixed & 18 & 8 & work \\
\hline 1 & focus group & female & 42 & mixed & 11 & 12 & UBS \\
\hline 1 & focus group & female & 43 & white & 15 & 2 & UBS \\
\hline 1 & focus group & female & 50 & black & 15 & 15 & UBS \\
\hline 2 & focus group & female & 57 & mixed & 8 & 17 & UBS \\
\hline 2 & focus group & female & 65 & mixed & 11 & 30 & UBS \\
\hline 2 & focus group & female & 74 & white & 5 & 20 & UBS \\
\hline 3 & focus group & male & 43 & black & 11 & 3 & UBS \\
\hline 3 & focus group & male & 65 & mixed & 8 & 8 & UBS \\
\hline 3 & focus group & male & 65 & white & illiterate & 15 & UBS \\
\hline 4 & focus group & female & 51 & mixed & 16 & 13 & Home \\
\hline 4 & focus group & female & 55 & white & 11 & 3 & Home \\
\hline 4 & focus group & female & 56 & white & 16 & 1 & Home \\
\hline 1 & structured & male & 45 & white & 19 & 10 & work \\
\hline 2 & structured & female & 40 & white & 20 & 4 & work \\
\hline 3 & structured & female & 44 & white & 26 & 9 & work \\
\hline 4 & structured & male & 40 & white & 20 & 3 & public place \\
\hline
\end{tabular}

PROM: patient-reported outcome measure; UBS: unidade básica de saúde (primary healthcare clinic)

\section{Acknowledgements}

We thank the ACS (Agentes Comunitários de Saúde - Community Healthcare Agents) and all the team from UBS Jardim Boa Vista and UBS Jardim D'Abril in São Paulo and Paul Jacob Grandjean-Thomsen, Penha de Fátima M. Maia Nielsen, Adriana Machado and Paula Fernanda C. Abrão who worked as translators during the bilingual panel. We also thank Susanne Reventlow for all the support during the qualitative content analysis.

\section{Authors' contributions}

$J G, J B$ and $A O$ designed the study. JG conducted the literature review. JG, LB and $\mathrm{AO}$ posted an invitation on Facebook in order to recruit suitable informants. JG had telephone contact with the informants.

$J G$ and JB (via skype) conducted the dual panel sessions. JG was present and conducted the panels in person in Brazil and facilitated the group process and mediated discussions when necessary. JB was available via Skype to discuss possible interpretations of the original content.

JG conducted all the single interviews and conducted the focus group interviews together with $L B$. JG, JB, LB and $A O$ conducted the data analysis and generated new items. JG drafted the manuscript. $A O$ and JB contributed to revisions with important intellectual content. All authors accepted the final version of the manuscript. All authors had full access to all data in the study and take responsibility for the integrity of the data and the accuracy of the data analysis. JG is guarantor.

\section{Authors' information}

All authors are clinicians in General Practice. However, none of the informants were patients in our clinics. JG and LB had no previous experience with qualitative research. $A O$ and $J B$ are experienced researchers in qualitative methods and, respectively, supervisor and co-supervisor of JG in his PhD thesis. An English ad hoc translation of the empirical material was shared with JB, who does not read or speak Brazilian Portuguese. JB is Danish and JG, LB and $A O$ are Brazilians.

\section{Funding}

The first author was granted a 1 year scholarship by CAPES (Brazil) for completing a 1 year visit to Københavns Universitet during his Ph.D. programme in Brazil. Award number: 88881.132103/2016-01.

\section{Availability of data and materials}

The datasets used and/or analyzed during the current study are available from the corresponding author on reasonable request. 


\section{Ethics approval and consent to participate}

All informants provided their informed consent, and the study was approved by the Faculdade de Medicina da Universidade de São Paulo ethical committee, CAAE 54699716.0.0000.0065.

\section{Consent for publication}

Not applicable.

\section{Competing interests}

The authors declare that they have no competing interests.

\section{Author details}

${ }^{1}$ Departamento de Medicina Preventiva, Faculdade de Medicina da Universidade de São Paulo, Av. Dr Arnaldo, 45520 andar. CEP, São Paulo, SP 01246-903, Brazil. ${ }^{2}$ Hospital das Clínicas da Faculdade de Medicina da Universidade de São Paulo, Rua Dr. Ovídio Pires de Campos, 225. CEP, São Paulo, SP 05403-010, Brazil. ${ }^{3}$ Forskningsenheden for Almen Praksis, Center for Sundhed og Samfund, Københavns Universitet, Øster Farimagsgade, Bygning 24. Postboks 2099, 1014 København K, Denmark.

Received: 19 June 2019 Accepted: 11 December 2019

Published online: 31 December 2019

\section{References}

1. Malachias, M. V. B., Gomes, M. A. M., Nobre, F., Alessi, A., Feitosa, A. D., \& Coelho, E. B. (2016). 7th Brazilian guideline of arterial hypertension: Chapter 2 - diagnosis and classification. Arquivos Brasileiros de Cardiologia, 107(3 Suppl 3), 7-13.

2. Harris, R. P., Sheridan, S. L., Lewis, C. L., Barclay, C., Vu, M. B., Kistler, C. E., et al. (2014). The harms of screening: A proposed taxonomy and application to lung cancer screening. JAMA Internal Medicine, 174(2), 281-285.

3. Heleno, B., Siersma, V., \& Brodersen, J. (2015). Waiting time and the psychosocial consequences of false-positive mammography: Cohort study. Journal of Negative Results in Biomedicine, 14(1), 8.

4. Kaczorowski, J., Chambers, L. W., Dolovich, L., Paterson, J. M., Karwalajtys, T., Gierman, T., et al. (2011). Improving cardiovascular health at population level: 39 community cluster randomised trial of cardiovascular health awareness program (CHAP). BMJ., 342, d442.

5. Wilt, T. J., Kansagara, D., \& Qaseem, A. (2018). Physicians ftCGCotACo. Hypertension limbo: Balancing benefits, harms, and patient preferences before we lower the Bar on blood pressure. Annals of Internal Medicine, 168(5), 369-370.

6. Bell, K. L., Doust, J., \& Glasziou, P. (2018). Incremental benefits and harms of the 2017 american college of cardiology/american heart association high blood pressure guideline. JAMA Internal Medicine.

7. Krumholz, H. M. (2018). Blood pressure guidelines as starting point in clinical decisions. BMJ., 360, j5862.

8. Pater, C. (2005). The blood pressure "uncertainty range" - a pragmatic approach to overcome current diagnostic uncertainties (II). Current Controlled Trials in Cardiovascular Medicine, 6(1), 5.

9. Myers, M. G., Kaczorowski, J., Paterson, J. M., Dolovich, L., \& Tu, K. (2015). Thresholds for diagnosing hypertension based on automated office blood pressure measurements and cardiovascular risk. Hypertension., 66(3), 489-495.

10. Ram, C. V., \& Giles, T. D. (2010). The evolving definition of systemic arterial hypertension. Curr Atheroscler Rep, 12(3), 155-158.

11. Haase, C. B., Gyuricza, J. V., \& Brodersen, J. (2019). New hypertension guidance risks overdiagnosis and overtreatment. BMJ., 365, I1657.

12. Sheppard, J. P., \& Stevens, S. (2018). Stevens R, et al. JAMA Internal Medicine: Benefits and harms of antihypertensive treatment in low-risk patients with mild hypertension.

13. Diao, D., Wright, J. M., Cundiff, D. K., \& Gueyffier, F. (2012). Pharmacotherapy for mild hypertension. The Cochrane database of systematic reviews, 8 , CD006742.

14. Glasziou, P. P., \& Irwig, L. M. (1995). An evidence based approach to individualising treatment. Bmi., 311(7016), 1356-1359.

15. Shamsi, A. P., Dehghan Nayeri, N. P., \& Esmaeili, M. P. (2017). Living with hypertension: A qualitative research. International Journal of Community Based Nursing and Midwifery, 5(3), 219-230.
16. de Castro, V. D., \& Car, M. R. (2000). Daily living with hypertension: Changes, restrictions and reactions. Revista da Escola de Enfermagem da USP, 34(2), 145-153.

17. Sangren, H., Reventlow, S., \& Hetlevik, I. (2009). Role of biographical experience and bodily sensations in patients' adaptation to hypertension. Patient Educ Couns, 74(2), 236-243.

18. Gyuricza JV, Brodersen J, Machado LBM, d'Oliveira AFPL. 'People say it is dangerous': Psychosocial consequences of Labelling People with Mild Hypertension: A Qualitative Study. Manuscript submitted for publication. BMC Fam Pract. 2019.

19. Alderman, M. H., \& Lamport, B. (1990). Labelling of hypertensives: A review of the data. Journal of Clinical Epidemiology, 43(2), 195-200.

20. Haynes, R. B., Sackett, D. L., Taylor, D. W., Gibson, E. S., \& Johnson, A. L. (1978). Increased absenteeism from work after detection and labeling of hypertensive patients. New England Journal of Medicine, 299(14), 741-744.

21. Alderman, M. H., Charlson, M. E., \& Melcher, L. A. (1981). Labelling and absenteeism: The Massachusetts mutual experience. Clinical and Investigative Medicine, 4(3-4), 165-171.

22. Mossey, J. M. (1981). Psychosocial consequences of labelling in hypertension. Clin Invest Med, 4(3-4), 201-207.

23. Bloom, J. R., \& Monterossa, S. (1981). Hypertension labeling and sense of well-being. Am J Public Health, 71(11), 1228-1232.

24. Charlson, M. E., Alderman, M., \& Melcher, L. (1982). Absenteeism and labelling in hypertensive subjects. Prevention of an adverse impact in those at high risk. Am J Med, 73(2), 165-170.

25. Soghikian, K., Fallick-Hunkeler, E. M., Ury, H. K., \& Fisher, A. A. (1981). The effect of high blood pressure awareness and treatment on emotional wellbeing. Clin Invest Med., 4(3-4), 191-196.

26. Trevisol, D. J., Moreira, L. B., Kerkhoff, A., Fuchs, S. C., \& Fuchs, F. D. (2011). Health-related quality of life and hypertension: A systematic review and metaanalysis of observational studies. Journal of Hypertension, 29(2), 179-188.

27. Streiner DL, Norman GR, Cairney J. Health measurement scales: a practical guide to their development and use. Fifth edition. ed. Oxford: Oxford University Press; 2015. xiii, 399 pages P.

28. McKenna, S. P., \& Doward, L. C. (2004). Integrating patient-reported outcomes. Value Health, 7(Suppl 1), S9-S12.

29. Wiebe, S., Guyatt, G., Weaver, B., Matijevic, S., \& Sidwell, C. (2003). Comparative responsiveness of generic and specific quality-of-life instruments. Journal of Clinical Epidemiology, 56(1), 52-60.

30. Wan, C., Jiang, R., Tu, X. M., Tang, W., Pan, J., Yang, R., et al. (2012). The hypertension scale of the system of quality of life instruments for chronic diseases, QLICD-HY: A development and validation study. International Journal of Nursing Studies, 49(4), 465-480.

31. Roca-Cusachs A, Ametlla J, Calero S, Comas O, Fernandez M, Lospaus R, et al. [Quality of life in arterial hypertension]. Medicina Clinica (Barc). 1992; 98(13):486-90.

32. Dalfó i Baqué, A., Badia i Llach, X., \& Roca-Cusachs, A. (2002). Cuestionario de calidad de vida en hipertensión arterial (CHAL). Atención Primaria, 29(2), 116-121.

33. Badia, X., Roca-Cusachs, A., Dalfo, A., Gascon, G., Abellan, J., Lahoz, R., et al. (2002). Validation of the short form of the Spanish hypertension quality of life questionnaire (MINICHAL). Clinical Therapeutics, 24(12), 2137-2154.

34. Brodersen J, Doward LC, Thorsen H, McKenna SP. Writing Health-Related Items for Rasch Models - Patient-Reported Outcome Scales for Health Sciences: From Medical Paternalism to Patient Autonomy. In: Christensen KB, Kreiner S, Mesbah M, editors. Rasch Models in Health. Applied Mathematics Series. London, UK: ISTE Ltd.; 2013. p. 281-98.

35. Doward, L. C., \& McKenna, S. P. (2004). Defining patient-reported outcomes. Value Health, 7(Suppl 1), S4-S8.

36. Soutello, A. L., Rodrigues, R. C., Jannuzzi, F. F., Sao-Joao, T. M., Martinix, G. G., Nadruz Jr., W., et al. (2015). Quality of life on arterial hypertension: Validity of known groups of MINICHAL. Arquivos Brasileiros de Cardiologia, 104(4), 299-307.

37. Brodersen, J., \& Thorsen, H. (2008). Consequences of screening in breast Cancer (COS-BC): Development of a questionnaire. Scandinavian Journal of Primary Health Care, 26(4), 251-256.

38. Brodersen, J., Thorsen, H., \& Kreiner, S. (2010). Consequences of screening in lung Cancer: Development and dimensionality of a questionnaire. Value Health, 13(5), 601-612.

39. Brodersen, J., Siersma, V., \& Thorsen, H. (2018). Consequences of screening in cervical cancer: Development and dimensionality of a questionnaire. BMC Psychology, 6(1), 39. 
40. Brodersen, J., Hansson, A., Johansson, M., Siersma, V., Langenskiöld, M., \& Pettersson, M. (2018). Consequences of screening in abdominal aortic aneurysm: Development and dimensionality of a questionnaire. Journal of Patient-Reported Outcomes, 2(1), 37.

41. Bolejko, A., Wann-Hansson, C., Zackrisson, S., Brodersen, J., \& Hagell, P. (2013). Adaptation to Swedish and further development of the 'Consequences of screening - breast Cancer' questionnaire: A multimethod study. Scandinavian Journal of Caring Sciences, 27(2), 475-486.

42. Swaine-Verdier, A., Doward, L. C., Hagell, P., Thorsen, H., \& McKenna, S. P. (2004). Adapting quality of life instruments. Value Health, 7(Suppl 1), S27-S30.

43. Someren M, Barnard Y, Sandberg J. The Think Aloud Method - A Practical Guide to Modelling CognitiveProcesses 1994.

44. McKenna, S. P., \& Doward, L. C. (2005). The translation and cultural adaptation of patient-reported outcome measures. Value Health, 8(2), 89-91.

\section{Publisher's Note}

Springer Nature remains neutral with regard to jurisdictional claims in published maps and institutional affiliations.

\section{Submit your manuscript to a SpringerOpen ${ }^{\circ}$ journal and benefit from:}

- Convenient online submission

- Rigorous peer review

- Open access: articles freely available online

High visibility within the field

- Retaining the copyright to your article

Submit your next manuscript at $\boldsymbol{\nabla}$ springeropen.com 